\title{
Apoyo al sistema político y tolerancia política en el periodo gubernamental 2016-2020 a través de la identidad étnica
}

\author{
Edwin Cohaila \\ Universidad Antonio Ruiz de Montoya
}

\section{Resumen}

El Perú ha atravesado, en este período gubernamental que empezó el 2016, diferentes situaciones políticas, la renuncia del presidente Kucyznski, la asunción al mando por parte de su vicepresidente Martín Vizcarra, el cierre del Congreso, la prisión preventiva de muchos actores políticos; sin embargo, se continuó con el régimen democrático, lo que podría suponer que la población mantiene un apoyo al sistema político y una tolerancia política, puesto que todo se encauzó dentro del marco constitucional. Para averiguar esta situación, se analizará la data que provee el Barómetro de las Américas (LAPOP) para los años 2016/2017 y 2018/2019 para el caso peruano, pero haciendo notar si existe alguna diferencia entre ambos periodos según la identidad étnica. El análisis manifiesta que el apoyo al sistema político se ha mantenido sin variación, mientras que la tolerancia política se ha incrementado; no obstante, al interior de la identidad étnica no todos los grupos se han mantenido bajo esa misma línea, ya que se observan diferencias significativas en especial en el grupo étnico quechua y mestizo.

Palabras clave: apoyo al sistema político, tolerancia política, identidad étnica, opinión pública, cultura política

\begin{abstract}
:
Peru has gone through this governmental period that began in 2016 with different political situations, resignation of President Kucyznski, assumption of command by his vice president Martín Vizcarra, closure of Congress, preventive detention of many political actors; however, the democratic regime continued, which could suppose that the population maintains support for the political system and political tolerance, since everything was channeled within the constitutional framework. To find out this situation, the data provided
\end{abstract}


by the Latin American Public Opinion Project for the years 2016/2017 and 2018/2019 will be analyzed for the Peruvian case, but noting if there is any difference between the two periods according to ethnic identity. The analysis shows that support for the political system has remained unchanged, while political tolerance has increased, although within ethnic identity not all groups have remained along the same line, since there are significant differences especially in the quechua and mestizo ethnic group.

Keywords: support for the political system, political tolerance, ethnic identitiy, public opinon, political culture

\section{Introducción}

El Perú ha atravesado diferentes acontecimientos en el ámbito político en este periodo gubernamental que empezó el 2016 y que aún no termina ${ }^{1}$. Después de una segunda vuelta muy reñida, en la que el candidato Pedro Pablo Kuczynski ganó con apenas el 50,14 \% de los votos válidos a la candidata Keiko Fujimori del partido Fuerza Popular, quien había conseguido la mayoría de congresistas en un poder legislativo unicameral como en el Perú, se sucedieron diferentes acontecimientos, como la renuncia del presidente Kuczynski, la asunción de su vicepresidente Martín Vizcarra, la confrontación existente entre los congresistas de Fuerza Popular con el recién instaurado gobierno, el proceso de referéndum, el cierre del Congreso, es decir, diferentes hechos que hacían tambalear al gobierno; no obstante, a pesar de estos avatares, el régimen democrático se mantuvo, lo que podría corroborar la existencia de un incremento de apoyo al sistema político en general y una tolerancia política de sus ciudadanos.

Para averiguar lo anterior, se analiza, a partir de los constructos de los índices de apoyo al sistema político y a la tolerancia política que ofrece las bases de datos del Barómetro de las Américas (LAPOP por sus

1 El actual periodo concluye el 28 de julio de 2021. 
siglas en inglés) aplicados en los años 2016/2017 y 2018/2019, es decir, en todo el proceso político que ha atravesado el Perú últimamente. No obstante, lo que se desea averiguar, además, es cómo han sido valorados estos índices según los grupos étnicos en el país.

Por ello, se plantea como hipótesis que, en este periodo, si bien ha habido un mismo nivel de apoyo al sistema político, es posible advertir cambios significativos al interior de los grupos étnicos. Del mismo modo, se plantea que la tolerancia política ha sufrido un incremento en este periodo, pero no se refleja al interior de los grupos étnicos.

Se observa que el apoyo al sistema político se ha mantenido similar en todos los grupos étnicos, a excepción del grupo quechua, en el que se observa un decrecimiento en este apoyo al sistema. Por el lado de la tolerancia política, se observa un incremento en dicha tolerancia de manera general, pero que al interior de los grupos étnicos no ha habido cambio alguno, a excepción del grupo mestizo, en el cual esta diferencia es significativa.

\section{Contexto político}

El proceso electoral de 2016 culminó con una segunda vuelta electoral entre los candidatos Pedro Pablo Kuczynski (PPK) del partido político Peruanos por el Cambio y la candidata de Fuerza Popular, Keiko Fujimori. Si bien la candidata Keiko Fujimori había obtenido la primera fuerza parlamentaria ( 73 congresistas de un total de 130 a nivel nacional), no había podido alcanzar la mitad de los votos válidos más uno como norma el Jurado Nacional de Elecciones, ente encargado del proceso normativo electoral peruano; por tanto, tenía que realizarse una segunda vuelta con el candidato que había obtenido la segunda preferencia electoral; es allí donde PPK gana la elección.

El partido de PPK solo obtuvo 18 congresistas para dicho período ${ }^{2}$, situación que se vio prontamente sacudida por los constantes pedidos del partido Fuerza Popular que solicitaban ante cualquier incidente

2 Los otros partidos tuvieron la siguiente cantidad de congresistas electos: Frente Amplio (20), Alianza para el Progreso (9), Acción Popular (5), Apra (5). 
político las explicaciones del ministro encargado; es así como ante las presuntas compras irregulares de computadoras y la demora en la implementación de obras para los Juegos Panamericanos Lima 2019, el ministro Jaime Saavedra fue censurado por el Congreso en diciembre de $2016^{3}$.

A mediados de noviembre de 2017, Kuczynski manifiesta en un mensaje a la Nación que él no había realizado consultorías con su empresa consultora Westfield Capital para la Constructora Odebrecht cuando era ministro, sino cuando dejó de serlo y que tampoco había recibido dinero para su campaña política. En este mensaje, estuvo agobiado por las investigaciones del Equipo Especial de la Fiscalía denominado Lava Jato; sin embargo, el 13 de diciembre, la misma empresa constructora confirma pagos a la Consultora de Kuczynski ${ }^{4}$. Días después, el Congreso plantea debatir la destitución por "incapacidad moral permanente". El 21 de diciembre, PPK supera la moción de destitución (se tenía que obtener 87 votos, mayoría calificada) gracias a que el congresista Kenji Fujimori y otros diez congresistas no siguieron las consignas de su partido y apoyaron al mandatario. Antes de la Navidad de 2017, PPK promulga un indulto humanitario a Alberto Fujimori, que reflejaba en la opinión pública que se estaba "pagando" un favor por el apoyo del hijo en la moción de destitución ${ }^{5}$.

3 Esta censura por parte del Congreso fue criticada por varios sectores, para el excongresista Daniel Mora, obedecía principalmente a las reformas educativas impulsadas por este sector que afectaban a grupos de poder y económicos (ver: https://rpp.pe/politica/gobierno/asi-de-claro-por-que-jaime-saavedra-corre-elriesgo-de-ser-censurado-noticia-1016751) No hay que olvidar tampoco que Jaime Saavedra era ministro de Educación desde el 2013 y había sido el único ministro que continuó después del cambio de gobierno.

4 Odebrecht confirmó el pago de más de setecientos mil dólares entre los años 2004 y 2007.

5 Hubo demasiadas críticas al indulto. Algunos ministros renunciaron, como Carlos Basombrio y Salvador del Solar. La Coordinadora Nacional de Derechos Humanos presentó una denuncia ante la Corte Internacional de Derechos Humanos, la cual recomendó que esta demanda fuera tratada en el orden judicial peruano y ceñirse a lo establecido frente a la situación de los procesos de indulto; tiempo después, la Corte Suprema ordenó la vuelta a prisión de Alberto Fujimori. 
El 8 de marzo se presenta una nueva moción de destitución, en la que se aludía a determinadas "compras" de congresistas de la oposición para que apoyasen al gobierno a cambio de determinadas obras en las regiones donde estos congresistas habían sido electos. Es así que, semanas después, se revelan videos en los cuales los ministros del gobierno ofrecen determinadas obras en el contexto previo a esta votación de vacancia presidencial. Al día siguiente de la propagación de estos videos, el 21 de marzo de 2018, el presidente Kuczynski presenta su renuncia al cargo ${ }^{6}$.

En ese contexto, asume el poder Martín Vizcarra, quien, en su mensaje presidencial de 28 de julio de 2018, anuncia una reforma política que será evaluada en un referéndum en diciembre de ese año. Esta reforma incluía cuatro aspectos: la conformación de una Junta Nacional de Justicia, la normativa sobre el financiamiento de partidos, la no reelección inmediata de congresistas y el establecimiento de la bicameralidad ${ }^{7}$.

Entre los meses que siguieron hasta antes del referéndum pactado para el 9 de diciembre, hubo muchas tensiones entre el Congreso y el Ejecutivo; tan es así que el presidente del Consejo de Ministros presentó una cuestión de confianza que fue aprobada para que se discutieran los temas planteados por el presidente en el mensaje a la Nación del 28 de julio. Si bien en el referéndum de diciembre de 2018 se aprobó mayoritariamente, por parte de la población, con la propuesta del presidente $^{8}$, el proceso en el Congreso era dilatar el reglamento y la aprobación de las normativas que la población había aprobado.

6 En la actualidad, PPK se encuentra bajo arresto domiciliario.

7 En este contexto, antes de su primer mensaje a la Nación, salen a la luz casos relacionados a jueces supremos y fiscales, lo que se ha denominado el Caso de los Cuellos Blancos.

8 La propuesta en referéndum que indicaba la bicameralidad no fue respaldada por el presidente y tampoco por la población, ya que los congresistas indicaban previamente que en el Congreso iban a reglamentar que un excongresista en el actual periodo podía postulara al Senado, lo que contradecía la propuesta de no reelección. 
Este primer año de gobierno de Vizcarra estuvo marcado, además, por las investigaciones de la Fiscalía Especial del Caso Lava Jato que, con información de las declaraciones recogidas desde Brasil implicaban casos de corrupción a expresidentes y principales líderes políticos? ${ }^{9}$. Ante estas investigaciones, hubo desde el Congreso opiniones de varios congresistas ${ }^{10}$ que intentaban que el Acuerdo entre la Fiscalía y Odebrecht no se llegara a firmar por parte del Poder Judicial, mientras que el gobierno manifestaba abiertamente su respaldo al trabajo del Ministerio Público ${ }^{11}$.

En esta tensión permanente con citaciones a varios ministros al hemiciclo, y con poco avance en la reglamentación de normativas establecidas en el referéndum, el presidente Vizcarra, en el mensaje a la Nación del 28 de julio de 2019, manifiesta un adelanto de las elecciones para el año 2020, lo que establecía un recorte en el mandato presidencial y en el de los congresistas. Los meses siguientes fueron de mucha tensión política hasta que el 30 de setiembre, en un mensaje a la Nación, el presidente Vizcarra establece el cierre del Congreso y convoca a elecciones para enero de 2020, debido a la negación de la confianza fáctica del presidente del Consejo de Ministros ${ }^{12}$, por lo que se pudo gobernar bajo decretos de urgencia hasta la nueva elección del Congreso que se dio en el plazo determinado el 2020.

9 Entre los casos, se puede mencionar a los expresidentes Alejandro Toledo, Ollanta Humala y Alan García; entre los líderes políticos, a Keiko Fujimori.

10 Desde Fuerza Popular, hubo muchos congresistas, como Luz Salgado y Lourdes Alcorta; desde Acción Popular, Víctor Andrés Belaúnde.

11 Basta solo recordar el apoyo brindado por la mayoría de los congresistas de Fuerza Popular al fiscal de la Nación, Pedro Chavarry, envuelto en audios de corrupción en el Caso Lava Jato.

12 El hecho puntual de la negación fáctica mencionada obedece a la solicitud del titular del Consejo de Ministros, Salvador del Solar, sobre la discusión de la forma de elección de los magistrados al Tribunal Constitucional, y al no haberse discutido previamente, se interpretó como una negación. Asimismo, al haberse negado previamente la confianza al Consejo de Ministros en el año 2017, encabezado por Fernando Zavala, se establecían dos negaciones al voto de confianza, lo que faculta constitucionalmente al cierre del Congreso. 
Como hemos señalado, en este periodo gubernamental que empezó el 2016, han sucedido varios acontecimientos políticos, que hacían dudar de que el Perú pudiera continuar bajo lo que establece la Constitución. Sin embargo, se ha mantenido dentro de los cauces democráticos, lo que podría manifestar cierta "estabilidad" en el apoyo al sistema de gobierno y una tolerancia política a grupos que tienen opiniones diferentes.

\section{Identificación étnica}

La construcción de la identidad étnica es un tema controversial, porque, como menciona Sulmont (2012), no existe un consenso de definición de pueblo indígena. No obstante, desde la construcción empírica, hay cierta coincidencia en que dicho concepto debe contener dimensiones como el idioma, las tradiciones culturales, el territorio y la autoidentificación (Sulmont, 2012).

Para el Instituto Nacional de Estadística e Informática (INEI), la autodefinición étnica involucra la forma como la persona se autopercibe, considerando para ello sus costumbres, antepasados y, además, si se siente parte de un grupo étnico.

Sin embargo, establecer un "grupo étnico" tampoco es ajeno a problemas, ya que dicho establecimiento supone que existen conglomerados donde las personas se encuentran de alguna forma delimitadas y sobre todo que son autoconscientes de dicha pertenencia (Brubaker 2004); por tanto, sería mejor entenderlo como algo no estático, sino como una categoría práctica, en términos relacionales, de procesos dinámicos (Brubaber 2004: 11).

Desde la parte más cuantitativa y considerando la premisa de Brubaker, existen dos formas de medir la idea de etnicidad o raza según Sulmont (2012). La primera utiliza categorías como lengua materna, lugar de origen, religión o color de piel, mientras que la segunda utiliza la lógica de que las personas se autoubiquen a través de categorías de tipo étnico o racial.

En el Censo de 2017, el último realizado por el INEI en el Perú, se utiliza por primera vez la categoría de autoidentificación étnica. 
El Barómetro de las Américas ha estado utilizando la pregunta de la autoidentificación y también la categoría del color de piel observada por el encuestador desde hace varios años.

A partir de estas formas de aproximarse a lo étnico, se han realizado diferentes estudios donde se analizan ciertas situaciones de estos grupos en el Perú; en algunos, se resalta ciertas diferencias o desigualdades desde el campo laboral y social.

Desde el campo laboral, el estudio de Moreno y otros (2012) quiso averiguar la existencia de discriminación racial para conseguir trabajo; para ello, utiliza modelos logit, donde se observa la probabilidad de conseguir trabajo, se hace notar que las personas clasificadas como blancas tienen un $100 \%$ de probabilidad de conseguir trabajo en comparación con una persona indígena, y una persona mestiza tiene un $30 \%$ más de probabilidad de conseguir trabajo que una persona indígena. En otro estudio, Galarza y otros (2012), a través de un estudio experimental a partir de los anuncios de empleo de un diario de circulación nacional, enviaron y armaron los documentos y le ańadieron una foto, y encontraron diferencias de medias significativas según el sexo de la persona y según lo "racial", ya que las mujeres andinas tenían menores posibilidades de ofertas de empleo que las mujeres blancas y que los hombres andinos.

El estudio de Valdivia (2003) encontró que poco más del $30 \%$ de encuestados se había sentido discriminado en su centro laboral. En otro estudio, Benavides y otros (2006) dan cuenta de que los jefes de hogar afroperuanos $(10,2 \%)$ se han sentido discriminados en sus centros de trabajo, debido principalmente a su color de piel y nivel socioeconómico. Del mismo modo, Yamada y otros (2011) encuentran diferencias significativas entre los ingresos de personas indígenas y no indígenas, y destacan como variable diferenciadora el nivel educativo de las personas.

Desde el campo de la salud, el estudio de Valdivia (2012), a través de técnicas cualitativas de observación y entrevistas en el Valle del Mantaro a personal de salud y pacientes en diferentes tipos de establecimientos de salud, encuentra un "paternalismo vertical" donde 
se conjugan una forma de trato con autoritarismo en la prestación del servicio por parte del personal médico. Para dicho autor, este trato refleja cierta discriminación étnica hacia mujeres indígenas.

Desde el ámbito educativo, Telles y Steele (2012) utilizan datos de LAPOP 2010 y encuentran cierta correlación entre el nivel educativo alcanzando (medido en años) y el color de piel; por tanto, las personas de color más claro de piel tienden a obtener mayores niveles educativos. Para dichos autores, el color de piel sigue siendo una forma de estratificación para la región latinoamericana. Por otro lado, Castro y otros (2012) analizaron las brechas educativas a partir de las diferencias étnicas y el sexo de la persona utilizando las encuestas de hogares del INEI. A partir de modelos estadísticos, concluyeron que la deserción educativa es mayor en los grupos étnicos quechua, aymara y afrodescendientes en relación con los grupos blanco/mestizo.

Como se ha observado, en diferentes campos de estudio, se nota cierta diferencia por el tema étnico y racial, marcado en el acceso a lo laboral y educativo principalmente, pero poco se ha estudiado a partir de ciertas categorías propias del campo político.

\section{Metodología}

El presente artículo analiza cómo ha transcurrido el apoyo al sistema político y a la tolerancia política en el actual periodo de gobierno en el Perú, habida cuenta de que han ocurrido cambios en el acontecer político.

Por tanto, partiendo desde los aconteceres políticos, se estima las siguientes preguntas de investigación:

P1: ¿Qué tanto ha cambiado el apoyo al sistema político a partir de los acontecimientos políticos en el actual periodo de gobierno según la identidad étnica?

P2: ¿Qué tanto ha cambiado la tolerancia política a partir de los acontecimientos políticos en el actual periodo de gobierno según la identidad étnica?

Las hipótesis planteadas son las siguientes: 
H1: El apoyo político no ha sufrido cambio alguno en el actual periodo de gobierno a partir de una medida general (media); sin embargo, al interior de la identificación étnica es posible advertir ciertos cambios en dicho apoyo en los grupos que se autoidentifican como quechua, aimara y negro.

H2: La tolerancia política no ha sufrido cambio alguno en el actual periodo de gobierno a partir de una medida general (media); sin embargo, al interior de la identificación étnica es posible advertir ciertos cambios en dicha tolerancia en los grupos que se autoidentifican como quechua, aimara y negro.

Ambos constructos son utilizados por el proyecto del Barómetro de las Américas para la elaboración de sus respectivos informes.

Para la construcción del apoyo al sistema político se utiliza un índice compuesto por cinco preguntas. Esta construcción recoge, como indica LAPOP, la propuesta de Booth y Seligson (2009), la cual manifiesta la creencia en la legitimidad de las instituciones políticas en un país y los niveles generalizados de apoyo a cómo está organizado el sistema político (Informe PERÚ LAPOP 2016-2017:118).

Para la tolerancia política, se utiliza un índice compuesto por cuatro preguntas, las cuales tratan de medir la tolerancia hacia los ciudadanos que tienen objeciones hacia el sistema político y se solicita si se aprueba que puedan tener ciertos derechos (Informe PERÚ LAPOP 20162017:120).

Para la identidad étnica, se ha utilizado la pregunta de autoidentificación étnica ${ }^{13}$.

El Barómetro de las Américas ha aplicado dos encuestas en este periodo, la del año 2016/2017 y en el año 2018/2019, situación precisa para comparar si ha existido variación entre estos índices según la identidad étnica.

13 Para mayor detalle ver Informe Perú LAPOP 2016-2017. 


\section{Resultados}

A partir de las preguntas de cada índice, se ha procedido, como indica LAPOP a su reconstrucción; después de ello, se ha procedido a convertir en una escala que toma valores de 0 a 100 puntos; por tanto, valores cercanos al 0 manifestaran nada de apoyo al sistema o nada de tolerancia política y valores más cercanos a 100 puntos manifestarán mucho apoyo al sistema o mucha tolerancia política ${ }^{14}$.

Así, tenemos los siguientes resultados para el apoyo al sistema político: como se puede observar en el Cuadro 1, el valor de la media el año 2016/2017 fue de 43,9 puntos, mientras que el año 2018/2019 fue de 41,9 puntos; a simple vista, uno podría suponer que el apoyo al sistema político ha descendido; sin embargo, al notar los intervalos de confianza al $95 \%$, se nota que ambos se transponen (es decir, que comparten valores); por tanto, se puede manifestar que el apoyo al sistema político se ha mantenido constante en este periodo de gobierno. No obstante, es preciso hacer notar que el apoyo al sistema político tampoco alcanza los cincuenta puntos, que sería el punto intermedio en este índice; por tanto, el apoyo al sistema político está por debajo de una puntuación intermedia.

\section{Cuadro 1. Apoyo al sistema político}

\begin{tabular}{|c|c|c|c|c|}
\hline \multirow{2}{*}{ Año } & \multirow{2}{*}{ Media } & \multirow{2}{*}{$\begin{array}{c}\text { Error } \\
\text { Estándar }\end{array}$} & \multicolumn{2}{|c|}{ Intervalo de Confianza al 95 \% } \\
\cline { 4 - 5 } & & Límite inferior & Límite superior \\
\hline $2016 / 2017$ & 43,99 & 0,40 & 43,20 & 44,78 \\
\hline $2018 / 2019$ & 41,96 & 0,54 & 40,91 & 43,03 \\
\hline
\end{tabular}

Fuente: LAPOP 2016/2017, 2018/2019

Elaboración propia

14 Igualmente, antes de proceder a trabajar con las bases de datos, se ha procedido a ponderarlas. Del mismo modo, antes de su conversión final, a las preguntas iniciales se ha procedido a calcular el Alpha de Cronbach para medir su fiabilidad y se obtuvieron valores superiores al 0,7 . Estos constructos obedecen a diferentes preguntas en escalas de puntaje diferenciados que después son promediados y convertidos en una escala de 0 a 100 puntos, donde 0 manifiesta nada del constructo (apoyo al sistema político y tolerancia política). Para mayor detalle, ver los informes LAPOP. https://www.vanderbilt.edu/lapop-espanol/publicaciones.php 
Al observar el índice de tolerancia política en ambos periodos analizados para el caso peruano, encontramos que el año de estudio 2016/2017 se tenía un puntaje de 47,74 puntos y que el año de estudio 2018/2019 se ha alcanzado un puntaje de 50,80 puntos. Observando las medias encontramos que la tolerancia política se ha incrementado, lo que se verifica con los intervalos de confianza, ya que estos no se trastocan (no comparten valores); por tanto, este incremento es significativo. Además, habrá que añadir que en este índice se ha pasado la línea intermedia del puntaje, aunque no por mucho.

\section{Cuadro 2. Tolerancia política}

\begin{tabular}{|c|c|c|c|c|}
\hline \multirow{2}{*}{ Año } & \multirow{2}{*}{ Media } & \multirow{2}{*}{$\begin{array}{c}\text { Error } \\
\text { Estándar }\end{array}$} & \multicolumn{2}{|c|}{ Intervalo de Confianza al 95 \% } \\
\cline { 4 - 5 } & & Límite inferior & Límite superior \\
\hline $2016 / 2017$ & 47,74 & 0,40 & 46,96 & 48,52 \\
\hline $2018 / 2019$ & 50,80 & 0,52 & 49,78 & 51,82 \\
\hline
\end{tabular}

Fuente: LAPOP 2016/2017, 2018/2019

Elaboración propia

Ahora bien, se va a analizar cada uno de los índices según la identidad étnica. En el índice de apoyo al sistema político, se puede observar que en todos los grupos étnicos se ha mantenido en similar situación; por tanto, siguen un mismo comportamiento en todo el periodo analizado, a excepción del grupo étnico quechua, en el que sí se observa una variación significativa, es decir, en este grupo étnico, el apoyo al sistema político sí se manifiesta con un decrecimiento, es decir, manifiestan menos apoyo al sistema político en estos años de estudio. 
Cuadro 3. Apoyo al sistema político según grupo étnico

\begin{tabular}{|c|c|c|c|c|c|}
\hline \multirow{2}{*}{ Año } & \multirow{2}{*}{$\begin{array}{l}\text { Grupo } \\
\text { Étnico }\end{array}$} & \multirow{2}{*}{ Media } & \multirow{2}{*}{$\begin{array}{c}\text { Error } \\
\text { Estándar }\end{array}$} & \multicolumn{2}{|c|}{$\begin{array}{c}\text { Intervalo de } \\
\text { Confianza al } 95 \% \\
\end{array}$} \\
\hline & & & & $\begin{array}{l}\text { Límite } \\
\text { inferior }\end{array}$ & $\begin{array}{l}\text { Límite } \\
\text { superior }\end{array}$ \\
\hline $2016 / 2017$ & Blanco & 44,54 & 1,14 & 42,29 & 46,78 \\
\hline $2018 / 2019$ & Blanco & 45,78 & 1,74 & 42,35 & 49,22 \\
\hline $2016 / 2017$ & Mestiza & 43,47 & 0,52 & 42,45 & 44,49 \\
\hline $2018 / 2019$ & Mestiza & 41,52 & 0,67 & 40,20 & 42,83 \\
\hline $2016 / 2017$ & Negra & 41,14 & 3,53 & 33,98 & 48,30 \\
\hline $2018 / 2019$ & Negra & 44,44 & 3,95 & 36,28 & 52,61 \\
\hline $2016 / 2017$ & Mulata & 45,67 & 3,10 & 39,32 & 52,02 \\
\hline $2018 / 2019$ & Mulata & 42,86 & 5,06 & 31,92 & 53,79 \\
\hline $2016 / 2017$ & Otra & 44,40 & 2,18 & 40,08 & 48,72 \\
\hline $2018 / 2019$ & Otra & 38,33 & 3,22 & 31,89 & 44,78 \\
\hline $2016 / 2017$ & Oriental & 53,78 & 5,65 & 40,95 & 66,60 \\
\hline $2018 / 2019$ & Oriental & 41,43 & 8,99 & 19,42 & 63,43 \\
\hline $2016 / 2017$ & Quechua & 47,00 & 1,16 & 44,72 & 49,27 \\
\hline $2018 / 2019$ & Quechua & 41,17 & 1,46 & 38,29 & 44,04 \\
\hline $2016 / 2017$ & Aymara & 40,39 & 2,47 & 35,45 & 45,32 \\
\hline $2018 / 2019$ & Aymara & 44,04 & 3,24 & 37,44 & 50,64 \\
\hline $2016 / 2017$ & $\begin{array}{l}\text { De la } \\
\text { Amazonia }\end{array}$ & 42,58 & 4,31 & 33,76 & 51,39 \\
\hline $2018 / 2019$ & $\begin{array}{l}\text { De la } \\
\text { Amazonia }\end{array}$ & 43,21 & 4,24 & 34,50 & 51,92 \\
\hline $2016 / 2017$ & Zamba & 51,34 & 2,74 & 45,74 & 56,95 \\
\hline $2018 / 2019$ & Zamba & 40,58 & 4,08 & 32,12 & 49,04 \\
\hline
\end{tabular}

Fuente: LAPOP 2016/2017, 2018/2019

Elaboración propia 
Este apoyo al sistema político de manera general, es decir, contemplando a todos los grupos étnicos, muestra niveles similares, pero al interior de estos se ha podido verificar cierta diferencia en el grupo quechua, mas no en el grupo aimara o en el negro como se presumía, ya que en estos se ha mantenido el mismo soporte al sistema político; no obstante, el hecho de que haya descendido en el grupo quechua no conlleva que todo este soporte al apoyo político descienda.

Este apoyo al sistema político es construido por cinco indicadores, los cuales manifiestan el respeto a las instituciones, el orgullo por el sistema político, la creencia de que los tribunales de justicia garantizan un juicio justo, la creencia de que los derechos básicos están protegidos y que se debería apoyar al sistema político. Al observar al interior del grupo quechua sobre el conjunto de estos indicadores, se puede notar que el decrecimiento se da en la creencia de que se debería apoyar al sistema político, mientras que en los otros indicadores se ha mantenido en similar proporción. Esta observación por parte del grupo étnico quechua resalta que este apoyo general pierde sustancia, tanto así que era uno de los indicadores con mayor puntaje en el ańo de estudio 2016/2017, lo que manifiesta cierta desazón por parte de este grupo, tal vez porque el sistema político no está resolviendo sus demandas o sus necesidades.

\section{Cuadro 4. Indicadores de apoyo al sistema según grupo étnico quechua}

\begin{tabular}{|l|c|c|c|c|c|}
\hline \multirow{2}{*}{ Indicador } & Año & Media & \multirow{2}{*}{$\begin{array}{c}\text { Error } \\
\text { Estándar }\end{array}$} & & \multicolumn{2}{c|}{$\begin{array}{c}\text { Intervalo de } \\
\text { Confianza al 95 \% }\end{array}$} \\
\cline { 5 - 7 } & & $\begin{array}{c}\text { Límite } \\
\text { inferior }\end{array}$ & $\begin{array}{c}\text { Límite } \\
\text { superior }\end{array}$ \\
\hline $\begin{array}{l}\text { Tribunales } \\
\text { garantizan juicio } \\
\text { justo }\end{array}$ & $2016 / 2017$ & 37,96 & 1,48 & 35,04 & 40,88 \\
\cline { 2 - 6 } & $2018 / 2019$ & 33,33 & 1,89 & 29,60 & 37,06 \\
\hline $\begin{array}{l}\text { Respeto a las } \\
\text { instituciones }\end{array}$ & $2016 / 2017$ & 50,94 & 1,61 & 47,77 & 54,11 \\
\cline { 2 - 6 } & $2018 / 2019$ & 45,17 & 1,96 & 41,31 & 49,03 \\
\hline
\end{tabular}




\begin{tabular}{|l|c|c|c|c|c|}
\hline \multirow{2}{*}{$\begin{array}{l}\text { Los derechos } \\
\text { básicos están } \\
\text { protegidos }\end{array}$} & $2016 / 2017$ & 43,10 & 1,58 & 40,00 & 46,21 \\
\cline { 2 - 6 } & $2018 / 2019$ & 36,60 & 1,81 & 33,04 & 40,17 \\
\hline \multirow{2}{*}{$\begin{array}{l}\text { Orgullo por el } \\
\text { sistema político }\end{array}$} & $2016 / 2017$ & 46,99 & 1,72 & 43,61 & 50,38 \\
\cline { 2 - 6 } & $2018 / 2019$ & 42,60 & 2,02 & 38,62 & 46,59 \\
\hline $\begin{array}{l}\text { Se debería } \\
\text { apoyar al sistema } \\
\text { político }\end{array}$ & $2016 / 2017$ & $\mathbf{5 7 , 6 4}$ & $\mathbf{1 , 6 1}$ & $\mathbf{5 4 , 4 8}$ & $\mathbf{6 0 , 7 9}$ \\
\cline { 2 - 6 } & $\mathbf{2 0 1 8 / 2 0 1 9}$ & $\mathbf{4 8 , 4 4}$ & $\mathbf{2 , 1 7}$ & $\mathbf{4 4 , 1 6}$ & $\mathbf{5 2 , 7 3}$ \\
\hline
\end{tabular}

Fuente: LAPOP 2016/2017, 2018/2019

Elaboración: Propia

En relación al índice de la tolerancia política, se observa que el comportamiento en ambos años ha sido similar en todos los grupos étnicos, a excepción del grupo mestizo en el que sí se observa un incremento significativo de la tolerancia política, y es el único grupo étnico que logra tener más de cincuenta puntos en dicho índice, es decir, se mantiene por encima del puntaje intermedio, tan es así que a partir de esta diferencia es que arrastra a todo el índice de manera general.

Cuadro 5. Tolerancia política según grupo étnico

\begin{tabular}{|c|l|c|c|c|c|}
\hline \multirow{2}{*}{ Año } & \multirow{2}{*}{$\begin{array}{c}\text { Grupo } \\
\text { Étnico }\end{array}$} & Media & \multirow{2}{*}{$\begin{array}{c}\text { Error } \\
\text { Estándar }\end{array}$} & \multicolumn{2}{c|}{$\begin{array}{c}\text { Intervalo de } \\
\text { Confianza al 95 \% }\end{array}$} \\
\cline { 5 - 7 } & & $\begin{array}{c}\text { Límite } \\
\text { inferior }\end{array}$ & $\begin{array}{c}\text { Límite } \\
\text { superior }\end{array}$ \\
\hline $2016 / 2017$ & Blanco & 48,33 & 1,14 & 46,08 & 50,58 \\
\hline $2018 / 2019$ & Blanco & 49,57 & 1,60 & 46,41 & 52,73 \\
\hline $2016 / 2017$ & Mestiza & 47,05 & 0,52 & 46,03 & 48,07 \\
\hline $2018 / 2019$ & Mestiza & 51,65 & 0,68 & 50,33 & 52,98 \\
\hline $2016 / 2017$ & Negra & 52,97 & 3,21 & 46,46 & 59,49 \\
\hline $2018 / 2019$ & Negra & 49,31 & 3,44 & 42,18 & 56,43 \\
\hline $2016 / 2017$ & Mulata & 51,58 & 4,30 & 42,78 & 60,38 \\
\hline
\end{tabular}




\begin{tabular}{|l|l|l|l|l|l|}
\hline $2018 / 2019$ & Mulata & 46,63 & 3,62 & 38,82 & 54,44 \\
\hline $2016 / 2017$ & Otra & 50,20 & 2,00 & 46,24 & 54,17 \\
\hline $2018 / 2019$ & Otra & 46,14 & 2,81 & 40,52 & 51,75 \\
\hline $2016 / 2017$ & Oriental & 49,39 & 6,97 & 33,56 & 65,23 \\
\hline $2018 / 2019$ & Oriental & 50,79 & 5,51 & 37,30 & 64,28 \\
\hline $2016 / 2017$ & Quechua & 49,29 & 1,08 & 47,17 & 51,42 \\
\hline $2018 / 2019$ & Quechua & 51,33 & 1,31 & 48,74 & 53,92 \\
\hline $2016 / 2017$ & Aymara & 45,33 & 2,46 & 40,41 & 50,24 \\
\hline $2018 / 2019$ & Aymara & 48,23 & 3,03 & 42,07 & 54,40 \\
\hline $2016 / 2017$ & $\begin{array}{l}\text { De la } \\
\text { Amazonia }\end{array}$ & 45,13 & 3,78 & 37,39 & 52,86 \\
\hline $2018 / 2019$ & $\begin{array}{l}\text { De la } \\
\text { Amazonia }\end{array}$ & 54,42 & 3,77 & 46,67 & 62,18 \\
\hline $2016 / 2017$ & Zamba & 45,62 & 4,57 & 36,28 & 54,96 \\
\hline $2018 / 2019$ & Zamba & 47,58 & 4,85 & 37,52 & 57,65 \\
\hline
\end{tabular}

Fuente: LAPOP 2016/2017, 2018/2019

Elaboración propia

Este incremento en la tolerancia política a nivel general no es propio de todos los grupos étnicos, sino solo del grupo mestizo que, por ser mayoritario, puede arrastrar a todo en su conjunto en su diferencia.

Al interior de este índice de tolerancia política compuesto por cuatro indicadores, como aprueba el derecho a votar a personas con diferente posición o ideas, aprueba el derecho de protestar, aprueba el derecho a estas personas con posición o ideas diferentes a ser candidato y aprueba que puedan elaborar un discurso, muestra que todos a excepción de aprueba el derecho a protestar es el único que se mantiene al mismo nivel en ambos años de estudio, aunque habrá que manifestar a su favor que es el indicador que ha tenido mayor tolerancia en todo este periodo de gobierno (superior a los sesenta puntos en dicha escala). 


\section{Cuadro 6. Indicadores de tolerancia política según grupo} étnico mestizo

\begin{tabular}{|c|c|c|c|c|c|}
\hline \multirow{2}{*}{ Indicador } & \multirow{2}{*}{ Año } & \multirow{2}{*}{ Media } & \multirow{2}{*}{$\begin{array}{c}\text { Error } \\
\text { Estándar }\end{array}$} & \multicolumn{2}{|c|}{$\begin{array}{c}\text { Intervalo de } \\
\text { Confianza al } 95 \%\end{array}$} \\
\hline & & & & $\begin{array}{l}\text { Límite } \\
\text { inferior }\end{array}$ & $\begin{array}{l}\text { Límite } \\
\text { superior }\end{array}$ \\
\hline \multirow{2}{*}{$\begin{array}{l}\text { Aprueba el } \\
\text { derecho a votar }\end{array}$} & $2016 / 2017$ & 50,78 & 0,70 & 49,41 & 52,15 \\
\hline & $2018 / 2019$ & 56,94 & 0,89 & 55,20 & 58,68 \\
\hline \multirow{2}{*}{$\begin{array}{l}\text { Aprueba el } \\
\text { derecho a } \\
\text { protestar }\end{array}$} & $2016 / 2017$ & 61,76 & 0,72 & 60,34 & 63,17 \\
\hline & $2018 / 2019$ & 63,94 & 0,87 & 62,23 & 65,65 \\
\hline \multirow{2}{*}{$\begin{array}{l}\text { Aprueba el } \\
\text { derecho a ser } \\
\text { candidato(a) }\end{array}$} & $2016 / 2017$ & 37,25 & 0,69 & 35,89 & 38,61 \\
\hline & $2018 / 2019$ & 41,56 & 0,88 & 39,82 & 43,29 \\
\hline \multirow{2}{*}{$\begin{array}{l}\text { Aprueba el } \\
\text { derecho a hacer } \\
\text { un discurso }\end{array}$} & $2016 / 2017$ & 38,35 & 0,70 & 36,98 & 39,72 \\
\hline & $2018 / 2019$ & 43,98 & 0,92 & 42,17 & 45,79 \\
\hline
\end{tabular}

Fuente: LAPOP 2016/2017, 2018/2019

Elaboración propia

\section{Conclusiones}

El apoyo al sistema político peruano se ha mantenido en similar proporción en todo el periodo de gobierno actual, aunque dicha puntuación no logra situarse al menos en un puntaje intermedio, sino por debajo de este. Los peruanos manifiestan que, a pesar de los acontecimientos políticos suscitados cambiantes y diferentes, creen aún en el sistema político y no se observa un revés en este índice. No obstante, al interior de los grupos étnicos, solo se observa en el grupo quechua un decrecimiento de este apoyo al sistema político; sin embargo, este decrecimiento observado no es capaz de concentrar o arrastrar a todo el índice; una de las razones es quizá que solo en un indicador de este índice es que se ha observado este decrecimiento, el cual denota la creencia de dar apoyo al sistema político peruano, debido quizás a que en este grupo étnico, al no observar que las desigualdades se 
van disminuyendo cree menos que este sistema político pueda apoyarlo y, por ello, no manifiesta un soporte determinado.

Por tanto, la primera hipótesis planteada se cumple a medias, ya que se había manifestado que el apoyo al sistema político se había mantenido, pero que al interior de los grupos étnicos, como el quechua, el aimara y el negro, se podía observar un decrecimiento, y solo se ha logrado observar esta diferencia en el grupo étnico quechua, mientras que el resto se mantiene en los mismos niveles de apoyo en todo el periodo gubernamental; por tanto, estos acontecimientos políticos no han suscitado ningún cambio en estos.

La tolerancia política que trata de medir cómo puede ser el comportamiento de tolerancia hacia otros grupos que tienen opiniones e ideas diferentes a uno muestra de manera general que se ha incrementado la tolerancia política, es decir, que estos acontecimientos políticos han suscitado en los ciudadanos a aprobar que estos grupos diferentes puedan también acceder a ciertos derechos. Al interior de los grupos étnicos, se puede observar que solo en el grupo mestizo ocurre un incremento significativo, mientras que en el resto se ha mantenido en similar proporción, es decir, que el grupo mestizo, por ser mayoritario, logra arrastrar todo este índice. No obstante, al desagregar los cuatro indicadores sobre lo que está compuesto la tolerancia política, se observa que en el grupo mestizo se da este incremento en todos los indicadores, a excepción del indicador sobre el derecho de protestar, aunque este nivel ha sido el de mayor puntaje en ambos años de estudio.

Por tanto, la segunda hipótesis planteada no se cumple, ya que se había manifestado que no había existido diferencia en la tolerancia política en ambos ańos de estudio en este actual periodo de gobierno. Asimismo, se había manifestado que al interior de los grupos étnicos se podía observar cierta diferencia en los grupos quechua, aimara y negro; no obstante, en ninguno de estos se observa diferencia alguna, sino más bien es en el grupo mestizo en el que se observa un incremento de la tolerancia política. 


\section{Referencias}

Benavides, M., Torero, M. y Valdivia, N. (2006). Pobreza, discriminación social e identidad: el caso de la población afrodescendiente en el Perú. En J. Stubbs y H. Reyes (eds.), Más allá de los promedios: afrodescendientes en América Latina. Washington D.C.: Banco Mundial.

Brubaker, R. (2004). Ethnicity Without Groups. Harvard: Harvard University Press.

Castro, J. F., Yamada, G. y Asmat, R. (2012). Diferencias étnicas y de sexo en el acceso y deserción en el sistema educativo peruano. En F. Galarza (ed.), Discriminación en el Perú: exploraciones en el Estado, la empresa y el mercado laboral (pp. 19-60). Lima: Universidad del Pacífico.

Galarza, F. y Yamada, G. (2012). Discriminación laboral en Lima: el rol de la belleza, la raza y el sexo. Documento de discusión. Lima: Universidad del Pacífico.

Galarza, F., L. Kogan y G. Yamada (2012). Detectando discriminación sexual y racial en el mercado laboral de Lima. En F. Galarza (ed.), Discriminación en el Perú: exploraciones en el Estado, la empresa y el mercado laboral (pp. 103-135). Lima: Universidad del Pacífico.

\section{LAPOP}

2018/2019 Informe preliminar Encuesta Perú. Universidad Vanderbilt

2016/2017 Cultura política de la democracia en Perú y las Américas. Universidad Vanderbilt

Moreno, M., Ñopo, H., Saavedra, J. y Torero, M. (2012). Detecting Gender and Racial Discrimination in Hiring through Monitoring Intermediation Services: The Case of Selected Occupations in Metropolitan Lima, Peru. World Development 40 (2), 315328. doi: 10.1016/j.worlddev.2011.05.003 
Santos, Martin (2014). La discriminación racial, étnica y social en el Perú: balance crítico de la evidencia empírica reciente. Debates en sociología, 39, 5-37

Sulmont, D. (2005). Encuesta nacional sobre exclusión y discriminación social. Informe final de análisis de resultados. Lima: Estudio para la Defensa y los Derechos de la Mujer.

(2012). Raza y etnicidad desde las encuestas sociales y de opinión: dime cuántos quieres encontrar y te diré qué preguntar. En C. Sanborn (ed.), La discriminación en el Perú (pp. 51-74). Lima: Universidad del Pacífico.

Telles, E. y Steele, L. (2012). Pigmentocracy in the Americas: How is Educational Attainment Related to Skin Color? Americas Barometer Insights, 73, 1-9.

Torero, M., Saavedra, J., Ñopo, H. y Escobal, J. (2004). An Invisible Wall? The Economics of Social Exclusion in Peru. En M. Buvinic, J. Mazza y R. Deutsch (eds.), Social Inclusion and Economic Development in Latin America (pp. 221-245). Washington D.C.: Inter-American Development Bank / Johns Hopkins University Press.

Valdivia, N., Benavides, M. y Torero, M. (2007). Exclusión, identidad étnica y políticas de inclusión social en el Perú: el caso de la población indígena y la población afrodescendiente. En Investigación, políticas y desarrollo en el Perú (pp. 603-655). Lima: Grade.

Valdivia, N. (2003). Etnicidad, pobreza y exclusión social: la situación de los inmigrantes indígenas en las ciudades de Cusco y Lima. En J. Uquillas, T. Carrasco y M. Rees (eds.), Exclusión social y estrategias de vida de los indígenas urbanos en Perú, México y Ecuador (pp. 29-129). Quito: Banco Mundial/Fideicomiso Noruego. 
(2012). La discriminación en el Perú y el caso de los servicios de salud: resultados de un estudio cualitativo en el Valle del Mantaro. En C. Sanborn (ed.), La discriminación en el Perú: balance y desafíos (pp. 85-111). Lima: Universidad del Pacífico.

Yamada, G., Lizarzaburu, A. y Samanamud, K. (2012). La persistencia de las brechas étnicas en el mercado laboral peruano. En F. Galarza (ed.), Discriminación en el Perú: exploraciones en el Estado, la empresa y el mercado laboral (pp. 61-101). Lima: Universidad del Pacífico. 